less cumbersome term of ischaemic atrophy. She used the term toxic atrophy for a glomerular lesion in chronic glomerulonephritis which is redescribed in more detail by Dr Meadows who puts forward the thesis that some cases of chronic glomerulonephritis develop as a progressive form of focal disease. In her Medical Research Council Special Report (No. 142), Russell (1929) had an interesting name for this concept-nephritis repens-which has never caught on. Perhaps these reports respose in dusty basements or galleries of libraries in Australia just as they do in this country.

The chapters on tubular disease are not quite as good as those on glomerular because of a lack of adequate integration with experimental work. In understanding the changes in biopsy and necrosy material from acute tubular necrosis, for example, it is essential to appreciate the appearances of regenerating tubular epithelium at various stages-the details of which are most easily seen in experimental mercury poisoning. The only consideration of such regeneration is confined to ischaemic tubular necrosis and the sequence is not brought out. The part which obstruction may play in the pathogenesis of tubular damage is not discussed; in this respect it is unfortunate that the paper of $\mathrm{By}$ waters (1946) in which he brought out the resemblance between changes in acute hydronephrosis and the crush syndrome has never received proper recognition.

J. F. SмITH

\section{Pictures of viruses}

Ultrastructure of Animal Viruses and Bacteriophages. An Atlas. Edited by A. J. Dalton and F. Hagenau. (Ultrastructure in Biological Systems vol. 5.) Pp. xii+413. (Academic (Harcourt Brace Jovanovich): New York and London, September 1973.) \$37.50.

THIs book contains a splendid collection of electron micrographs illustrating the structure and development of 65 animal viruses and bacteriophages. Though the text is variable from chapter to chapter it is mostly concisely informative. The index is very comprehensive but it did not include the Sendai virus, which is now much used in cell fusion studies.

The chapters are divided according to the virus groupings laid down by the International Committee on Nomenclature of Viruses (ICNV). Each begins with a concise account of the structure of the virion and of its intracellular replication. As might be expected the text is not a comprehensive treatise on each group of viruses nor is each group uniformly treated. For instance R. W. Horne and B. Roizman each devote nine pages to their respective groups the adenoviruses and the herpesviruses, whereas N. Higashi and A. J. Dalton fill only one page each about the togaviruses and the arenaviruses. Nevertheless all the chapters have useful lists of references.

Several authors have been particularly helpful by giving clear accounts of more obscure aspects of their chosen group. A. J. Dalton and F. Hagenau define the A, B and C particles found in neoplastic tissues and G. C. Goodman gives a detailed account of the cytopathic events in cells infected with picornavirus in the captions to the illustrations. J. L. Melnick gives an account of the efforts of the ICNV to name and classify the viruses.

It is a pity that a book which treats the animal viruses so fully should expend two chapters on bacteriophage but exclude the viruses of plants and invertebrates. But the book completely achieves its editors' aims, provides a valuable work of reference for the animal viruses and gives a concise view of the bacteriophages.

D. KAY

\section{Cosmic rays reach earth}

Cosmic Rays at Ground Level. Edited by A. W. Wolfendale. Pp. vii +232. (Institute of Physics: London and Bristol, 1973.) $£ 12.00$.

As a tribute to Professor G. D. Rochester on the occasion of his retirement, nine authors associated with the cosmic ray group at the University of Durham have chosen to write ten short reviews, in which they have summarised the present situation in experimental work on the fluxes of the various components of the secondary cosmic radiation detected at "ground level", in chapters on energetic muons (M. G. Thompson), protons and pions (Brooke), neutrons (Ashton), $\mu$ neutrinos (Osborne), e-neutrinos (E. C. M. Young), extensive air showers below $10^{17} \mathrm{eV}$ (Wdowczyk) and above $10^{17} \mathrm{eV}$ (Turver), and on searches for quarks and other hypothetical particles (Ashton). The work is not about the primary cosmic radiation, although for completeness a preliminary chapter (Wolfendale) sketches in some of its aspects. There is added a comprehensive account of the technique of observing trajectories of fast charged particles by neon flash tubes (Breare), a method used by most of the authors.

In several chapters the aim is principally to collect data on the particle fluxes near sea level, and the "ground" in the terms of reference strictly excludes mention of experiments at mountain altitudes: hence a theoretical framework for interpreting the observations is not the aspect of interest. In the circumstances it is natural that the authors, apart from collecting published data, present progress reports which tend to lean heavily for illustration on the experiments in which they and their colleagues took part, in the research groups founded by Rochester and Wolfendale; although extensive references are given to other experiments.

In the most substantial review, on observations of momentum spectra, angular distributions and charge ratios of energetic muons, readers with a peripheral interest in the subject may be surprised by the extent to which for many years results have depended on normalisation to an old experiment by Greisen: only recently has the normalisation been revised (by Allkofer and others), and it is useful to have this documented. (It is a pity though that the "ground level" rule bars any reference to the work of the Utah group.) Although some problems currently raised by the interpretation of the charge ratio are briefly introduced, the significance of the vertical momentum spectrum is not discussed. The reviewers of hadrons have very little to work with at energies above a few $\mathrm{GeV}$, mountain experiments having been more numerous, and the distinction between pions and protons (at sea level) evidently remains a problem. Here, though, a discussion of the method of deducing the energies of hadrons in the $\mathrm{TeV}$ range from the cascades they produce would have been welcome, as different experiments are almost certainly not using the same scale. (In the short chapter on neutrons, the method used to measure their spectrum is not mentioned at all.) And for all very high energy spectra, one would have liked to know how much accompanying particles might affect the measurements.

To observe neutrinos one has to go to deep mines, but with the excuse that the fluxes there and at sea level must be the same, Osborne gives a clear account of the three main experiments on $\mu$-neutrinos, and their significance. Young and Ashton have no positive observations to report on their exotic particles, but they indicate what intensities might reasonably be sought. In the articles on extensive air showers the emphasis shifts to illustrating progress being made by the authors and their colleagues in constructing models of shower development, which is a necessary preliminary to firm deductions about the energies and nature of the primary particles; although Turver also presents recent estimates of the energy spectrum, and isotropy, and recent work on the distribution of several particle components of air showers, largely from the Haverah Park experiments.

The fluxes of electrons, gamma rays and slow neutrons are not reviewed. It is a pity that the price is so high for a welcome but relatively short book.

A. M. Hillas 